\title{
Impaired performance of alpha7 nicotinic receptor knockout mice in the five-choice serial reaction time task
}

\author{
E. Hoyle • R. F. Genn • C. Fernandes • I. P. Stolerman
}

Received: 30 March 2006 / Accepted: 2 August 2006 / Published online: 20 September 2006

(C) Springer-Verlag 2006

\begin{abstract}
Rationale Nicotinic receptors have been implicated in attentional performance. Nicotine can improve attention in animals and humans, but knowledge about relevant receptor subtypes is very limited.

Objectives The aim was to examine the role of $\alpha 7$ receptors in attentional performance of mice and in effects of nicotine.

Materials and methods Mice with targeted deletion of the gene coding for the $\alpha 7$ subunit of nicotinic receptors and wild-type controls were trained on a five-choice serial reaction time task with food reinforcers presented under varying parametric conditions. Nicotine was administered in a range of doses $(0.001-1.0 \mathrm{mg} / \mathrm{kg} \mathrm{sc})$, including those reported to enhance attentional performance.

Results Initially the $\alpha 7^{-/-}$(knockout) mice responded less accurately and made more anticipatory responses. After task parameters were altered so that the time allowed for responding was reduced and anticipatory (impulsive) responses were punished by a time-out, the pattern of
\end{abstract}

E. Hoyle • R. F. Genn • I. P. Stolerman $(\bowtie)$

Section of Behavioural Pharmacology,

Institute of Psychiatry PO49, King's College London,

De Crespigny Park,

London SE5 8AF, UK

e-mail: i.stolerman@iop.kcl.ac.uk

C. Fernandes

Social, Genetic and Developmental Psychiatry Research Centre, Institute of Psychiatry, King's College London,

London, UK

Present address:

E. Hoyle

The Ness Foundation, Dochfour Business Centre,

Inverness IV3 8GY,

Scotland, UK performance deficits changed; there were increased omission errors in $\alpha 7^{-/-}$mice but normal levels of accuracy and anticipatory responding. Nicotine did not improve any measure of performance, either with the original training parameters or after retraining; the largest dose used $(1.0 \mathrm{mg} / \mathrm{kg})$ produced a general impairment of responding in $\alpha 7^{-/}$and wild-type mice.

Conclusions $\alpha 7$ nicotinic receptor knockout mice are impaired in performance of the 5-CSRTT, suggesting a possible role for $\alpha 7$ receptors in attentional processing. However, identification of a protocol for assessing attention-enhancing effects of nicotine in mice may require further modifications of test procedures or the use of different strains of animal.

Keywords Addiction - Schizophrenia - Nicotinic receptor Nicotine $\cdot$ Mice $\cdot$ Knockout $\cdot$ Drug abuse $\cdot$ Cognition .

Behavior $\cdot$ Attention

\section{Introduction}

Nicotinic acetylcholine receptors are pentameric cation channels, so-called because of the actions of nicotine in binding to these receptors and bringing about channel opening. Twelve subunits of neuronal nicotinic receptors have been identified, $\alpha 2-10$ and $\beta 2-4$. From these subunits the two most common nicotinic receptors formed in the brain are the heteromeric $\alpha 4 \beta 2$ and the homomeric $\alpha 7$ nicotinic receptors, at approximately 85 and $10 \%$ of total nicotinic receptors, respectively. The $\alpha 7$ nicotinic receptors are especially highly expressed in the hippocampus where, as well as mediating cholinergic neurotransmission, their high $\mathrm{Ca}^{++}$permeability (Seguela et al. 1993) engenders the capacity to modulate glutamatergic and GABAergic neuro- 
transmission both pre- and post-synaptically (Berg and Conroy 2002). This study aims to shed further light on the role of $\alpha 7$ receptors in behaviour and in the behavioural effects of nicotine.

Nicotine is the second most commonly abused drug in the world after alcohol; cigarette smoking, with its wellestablished risks of chronic disease, constitutes a great burden on societies worldwide. Considerable efforts have been expended in investigating the function of nicotinic receptors in nicotine addiction and in other neuropsychiatric conditions, and in mediating the cognitive effects of nicotine. For example, cigarette smoking is approximately twice as common in patients with schizophrenia, as it is within the general population. The $\alpha 7$ nicotinic receptor has been implicated in the pathogenesis of schizophrenia and more specifically in the P50 sensory gating deficit measurable in approximately $90 \%$ of schizophrenics, about $50 \%$ of their relatives and about $10 \%$ of control subjects (Waldo et al. 1991). The sensory gating deficit in schizophrenia is thought to reflect an input dysfunction that allows greater passage of information through the hippocampus for processing by other brain areas (Freedman et al. 2002). The density of $\alpha 7$ nicotinic receptors in hippocampus and cingulate cortex from schizophrenics is considerably reduced (Freedman et al. 1995; Marutle et al. 2001). It might, therefore, be expected that performance of tasks requiring sustained attention would require $\alpha 7$ nicotinic receptors.

Attempts to investigate the functional role of $\alpha 7$ nicotinic receptors at the behavioural level have most often involved the use of selective agonists and antagonists to these receptors. Another avenue of research that has recently become possible uses genetically modified mice with specific deletions of genes for neuronal nicotinic receptors. Mice with targeted deletion of the gene for the $\alpha 7$ nicotinic receptor lack $\alpha$-bungarotoxin binding sites in the hippocampus and fast, rapidly desensitizing nicotinic currents in hippocampal neurons (Orr-Urtreger et al. 1997). Viability and brain morphology appear normal in these mice; of special interest is the lack of any impairment of sensorimotor gating as measured with both auditory and tactile stimuli in the prepulse inhibition paradigm (Paylor et al. 1998). This result is consistent with the lack of improvement in prepulse inhibition with nicotinic $\alpha 7$ agonists (Olivier et al. 2001; Schreiber et al. 2002), although such agonists are able to improve auditory gating in animal models of sensory gating deficit (O'Neill et al. 2003).

In other behavioural studies, $\alpha 7^{-/-}$mice have not proved to be considerably different in behaviour from wild-type littermates (Paylor et al. 1998). In locomotion, $\alpha 7^{-/-}$mice had a tendency towards less horizontal and vertical activity and spent significantly more time in the centre of the open field than the wild-type mice, but in the light-dark test of anxiety there were no significant differences between the two groups (Paylor et al. 1998). In the delayed matching-toplace task in the Morris water maze, $\alpha 7^{-/-}$mice were slightly impaired in comparison with wild-types, as shown by a longer time to find the hidden platform (Fernandes et al. 2005). Nicotine-induced locomotor depression, hypothermia, seizures, drug discrimination and tolerance were unchanged in $\alpha 7^{-/-}$mice (Franceschini et al. 2002; Tritto et al. 2004; Stolerman et al. 2004; Naylor et al. 2005). However, the $\alpha 7^{-/-}$mice were less sensitive to impairments in contextual learning brought about by ethanol (Wehner et al. 2004).

The series of experiments presented in this paper use the five-choice serial reaction time task (5-CSRTT), a commonly used model of attentional performance that is considered analogous to the continuous performance test used in human research. The test requires responses to visual stimuli presented in one of five locations in one wall of an operant conditioning chamber. In a previous study Young et al. (2004) found that $\alpha 7^{-/-}$mice took longer to acquire the task than wild-type $\mathrm{C} 57 \mathrm{Bl} / 6 \mathrm{~J}$ mice and made more omission errors, but they were not different with respect to accuracy or the latencies of correct responses. Young et al. (2004) also reported significant improvements in accuracy and reductions in omission errors at several doses of nicotine in $\mathrm{C} 57 \mathrm{Bl} / 6 \mathrm{~J}$ mice, including a reduction in the latency of correct responses with a $0.3-\mathrm{mg} / \mathrm{kg}$ dose of nicotine. In this paper, the first published report of nicotine improving the performance of mice in the 5-CSRTT and the effects seen were not dose-related, but the findings were important and need replication and investigation under a wider range of conditions.

Several studies have reported that nicotine enhances the attentional performance of rats in the 5-CSRTT (Hahn and Stolerman 2002; Hahn et al. 2002, 2003a), although the use of selective nicotinic agonists and antagonists has provided no evidence for involvement of the $\alpha 7$ receptor. The relatively specific $\alpha 7$ agonist AR-R17779 had no effect upon task performance in an experimental procedure in which administration of nicotine, epibatidine and isoarecolone all improved performance (Hahn et al. 2003a). These agonists all have much higher relative affinities than ARR17779 for heteromeric nicotinic receptors. The $\alpha 4 \beta 2$ nicotinic agonist SIB1765F has also been shown to improve 5-CSRTT performance in rats (Grottick and Higgins 2000). The $\alpha 7$ antagonist methyllycaconitine did not block the effects of nicotine on response latency and anticipatory responding, whereas dihydro- $\beta$-erythroidine, an antagonist at heteromeric receptors, did block the effect of nicotine on these measures (Blondel et al. 2000; Grottick and Higgins 2000). However, the $\alpha 7$ agonist GTS-21 has been reported to improve performance in various tests of 
attention and memory in healthy human volunteers when given in repeated doses (Kitagawa et al. 2003) and in a test of memory in monkeys $24 \mathrm{~h}$ after administration (Briggs et al. 1997). The small number of drugs investigated and their limited selectivity indicates a need for investigations on the role of $\alpha 7$ receptors in attention by another approach, such as the $\alpha 7^{-/}$mouse.

The aims of the present study were, therefore, to investigate the attentional performance of $\alpha 7^{-/}$mice using the 5-CSRTT and to determine whether the gene deletion influenced any effects of nicotine on performance. If the $\alpha 7$ nicotinic receptor is important in attentional mechanisms then it should be possible to confirm the attentional deficits reported by Young et al. (2004) in $\alpha 7^{-/-}$mice. If nicotine improves attention, at least in part, through these receptors then it should also be possible to show that nicotineinduced attentional enhancement is diminished in such animals. The procedures used in the initial stages of training were based on those of Patel et al. (2006) because they were close to those used in studies of rats where nicotine improved performance (Hahn et al. 2002; Hahn and Stolerman 2002). Mice were also trained with both fixed and variable inter-trial intervals (ITI) so that $\alpha 7^{-/}$ and wild-type animals could be compared for their abilities in coping with the temporally unpredictable stimuli in the variable ITI condition. In the later stages of the study, some parameters were modified to make the training procedure more similar to that of Young et al. (2004) so as to facilitate comparisons and to clarify reasons for certain differences in the results obtained.

\section{Materials and methods}

Animals

The animals used were descendants of $\alpha 7^{-/-}$mice produced by Orr-Urtreger et al. (1997), bred from heterozygotes purchased from the Jackson Laboratory, USA (B6.129S7Chrna $7^{\mathrm{tm} 1}$ Bay , stock no. 003232). The mice were at backcross generation $\mathrm{N} 8$, the background strain was C57BL/6, donor strain 129S7 via ABI 2.1 ES cell line; subsequent breeding was always carried out using heterozygous pairs. Twenty-four male $\alpha 7^{-/-}$mice and 24 male wild-type littermates were used in initial training. Genotypes were determined with the methods used by Stolerman et al. (2004), and all mice were injected with TROVAN sterile transponder chips (Mid Fingerprint, Dorset UK) at age 10 weeks. These groups were divided into sub-groups of 12 mice each trained with fixed and variable ITI. One wild-type mouse was eliminated from the study at an early stage due to an unresolvable eye infection. Table 1 lists the subsequent parameter changes that took place as training proceeded.
Food restriction was started at age 11-12 weeks and behavioural training began a week later. The mice were kept at $80 \%$ of their free-feeding weights as calculated from a previously obtained growth curve for animals from the same colony. The animals were weighed daily and fed accordingly. All animals had access to water while in their home cages and were individually housed in a room at $21{ }^{\circ} \mathrm{C}$ and on a 12 -h light/dark cycle, with lights coming on at 0700 hours.

The studies complied with local ethical requirements and were carried out in accordance with the Animals (Experimental Procedures) Act, 1986.

\section{Apparatus}

Sound-insulated and ventilated enclosures containing aluminium operant chambers were used (CENES, Cambridge, UK). The chambers measured $14 \mathrm{~cm}$ across $\times 12 \mathrm{~cm}$ high $\times$ $11-13 \mathrm{~cm}$ deep. The back wall of the chamber was curved so that each nose-poke hole was equidistant from the feeder tray at the centre of the front of the chamber. There were five nosepoke holes $1 \mathrm{~cm}$ in diameter, $2 \mathrm{~cm}$ above the floor, $1.5 \mathrm{~cm}$ apart and $1.5 \mathrm{~cm}$ deep. Each hole had a green-light-emitting diode at the end and a photoelectric cell at the opening. The two house lights were situated $10 \mathrm{~cm}$ up the sides of the chamber. An Acorn computer running Arachnid software (Paul Fray, Cambridge, UK) under RISC OS collected the data.

\section{Training procedure}

The mice were trained for 5 days each week, Monday to Friday. The training procedure was based on that of Patel et al. (2006) and was modified from that of Humby et al. (1999) so as to resemble more closely the procedures in previous work carried out with the 5-CSRTT in rats (Bizarro et al. 2004). After the mice were habituated to handling, training began with four sessions of 5, 10, 15 and $15 \mathrm{~min}$ for the mice to habituate to the test chambers. The house lights were not illuminated at this stage. Before the next two sessions that were 15 min each in duration, 15-20 food pellets were placed in feeder trays before the start of each session. This was followed by two sessions of 15- and 20-min duration, respectively, in which food pellets were presented according to fixed-time schedules of 15 and $30 \mathrm{~s}$. Nose-poke training then started with four 30-min sessions of non-spatial training with the stimulus duration set at $60 \mathrm{~s}$ and with an ITI of $2 \mathrm{~s}$. As noted above, subgroups of mice were trained with ITI of fixed duration, whereas for other groups, individual ITI varied around the mean value (for details, see Table 1). In non-spatial training sessions, all five holes were illuminated simultaneously and a nose-poke in any hole was reinforced by delivery of a food pellet. For the first of these sessions, $25 \mathrm{mg}$ food pellets (PJPPP-0020, 
Table 1 Parameters for all experiments

\begin{tabular}{llllllll}
\hline Study & Stimulus duration & Limited hold & Fixed ITI & Variable ITI & Time-out & Anticipatory time-out & Sessions to criteria \\
\hline $1 \mathrm{a}$ & 60 & 0 & 2 & $2(0.25-3.75)$ & 0 & 0 & 7 \\
& 60 & 0 & 4 & $4(0.5-7.5)$ & 2 & 0 & 2 \\
& 10 & 0 & 4 & $4(0.5-7.5)$ & 2 & 0 & 2 \\
& 5 & 1 & 4 & $4(0.5-7.5)$ & 2 & 0 & 4 \\
$1 \mathrm{~b}$ & 1 & 4 & 4 & $4(0.5-7.5)$ & 2 & 0 & 28 \\
$2 \mathrm{a}$ & 1 & 5 & 4 & $4(0.5-7.5)$ & 2 & 0 & NA \\
$2 \mathrm{~b}$ & 1 & 5 & 4 & NA & 2 & 0 & 15 \\
\end{tabular}

Studies $1 \mathrm{a}$ and $2 \mathrm{a}$ refer to experiments looking at baseline (undrugged) performance at initial and final parameters, whereas studies $1 \mathrm{~b}$ and $2 \mathrm{~b}$ refer to the first and second of the experiments with nicotine. For experiment 1a, where ITI values are shown for both fixed and variable ITI mode, these refer to settings for different groups of mice. All times stated are in seconds. For variable ITI the mean, smallest and largest values are shown.

NA Not applicable

Sandown Chemical, UK) were placed at the front of each of the nose-poke holes to encourage the mice to nose-poke.

For spatial training, the house lights were on at all times except during time-outs. Each session lasted for $30 \mathrm{~min}$ and there was no limit to the number of trials in a session. A stimulus was randomly presented in one of the five holes and a response in the correct hole during the stimulus duration or the limited hold $(\mathrm{LH})$ period after the stimulus was reinforced and followed by a 10 -s allowance for the time taken to eat the food pellet. This was sufficient for mice to consume the food and return to attending to the task; the next ITI began at the end of the 10-s allowance for eating. In sessions in which there was a time-out, this was initiated by a nose-poke response into an incorrect hole during the stimulus duration or LH. Premature responses during the ITI in experiment 1 were recorded but had no programmed consequences. Timeouts involved turning off the lights for $2 \mathrm{~s}$ and were followed immediately by the start of the next ITI.

Experiment 1a: initial acquisition of attentional task

Table 1 describes the stages of training, with criteria for progression to the next stage being that the mean percentage of correct responses for each group was greater than $70 \%$ and the mean response latency for each group was less than the stimulus duration for two consecutive days. The training parameters were not advanced if the mice had not been trained the day before. Acquisition was considered to be complete when the mean percentages of correct responses was stable for two consecutive weeks.

Experiment 1b: first set of nicotine tests

For testing the effects of nicotine, a reduced number of 15 wild-type and $15 \alpha 7^{-/-}$mice were used because it was known from previous studies that this was a sufficiently large group size for assessing drug effects (Humby et al. 1999; Hahn and Stolerman 2002). The selected animals were those that had all been trained at a similar time of day previously and no other selection criterion was used. Up to this stage, some mice had been trained with fixed and some with variable ITI. All selected mice were switched to fixed ITI mode before drug testing started to reduce the number of independent variables; all other parameters remained unchanged. These mice were given saline injections subcutaneously in the flank after training on each of the 3 days before the first nicotine test day to allow habituation to the procedure to take place. There were four test days on consecutive Tuesdays and Fridays. Mice were allocated to a dosing schedule for injection of saline or $0.001,0.01$ or $0.1 \mathrm{mg} / \mathrm{kg}$ nicotine $10 \mathrm{~min}$ before the start of each test session; each mouse was tested once at each dose by the end of the study and dosing sequences were random. Young et al. (2004) found that these doses of nicotine significantly improved the task performance of nicotine-naïve mice.

Experiment 2a: retraining after changing parameters

Seven weeks after the end of experiment $1 b$, retraining of the same 30 mice commenced, followed by alterations of experimental parameters to make them resemble more closely those used by Young et al. (2004). The changes entailed reducing the LH from 5 to $2 \mathrm{~s}$, using variable ITI mode only, and including a 2-s time-out for anticipatory responses. House lights were extinguished during the timeouts during which no stimuli were presented, and the presentation of the next stimulus was delayed by the duration of the time-out. Although mice originally trained with fixed ITI mode were at this time trained exclusively in variable ITI mode, there were no differences in performance as a function of this variation in training history. Table 1 lists the details of all the changes in task parameters. The animals 
were trained under the altered parameters for 7 weeks, by which time performance was relatively stable. One $\alpha 7^{-/-}$ mouse developed an unusual behavioural phenotype. This mouse was markedly hyperactive in its home cage, required approximately twice as much food as the other animals to maintain its weight and made anticipatory responses at a rate 5-10 times greater than the next most impulsive mouse. No data from this mouse are presented.

\section{Experiment 2b: second set of nicotine tests}

A second experiment with nicotine began immediately after the end of experiment $2 \mathrm{a}$. The mice were injected with saline for 3 days before the start of the test sessions so that they might habituate to the injection procedure. There were eight test sessions on consecutive Tuesdays and Fridays. Mice were allocated to a dosing schedule for injection of saline or $0.001,0.003,0.01,0.03,0.1,0.3$ or $1 \mathrm{mg} / \mathrm{kg}$ nicotine $10 \mathrm{~min}$ before the start of each test session. Each mouse was tested once at each dose by the end of the study and dosing sequences were random.

Data analyses

The six behavioural measures selected for analysis were as follows:

- Accuracy (percentage of correct responses): $100 \times$ correct responses/(correct + incorrect responses)

- Omissions (percentage of omission errors): $100 \times$ omission errors/stimuli presented

- Latency of correct responses: the mean time between stimulus onset and a nose-poke in the correct hole

- Latency of incorrect responses: the mean time between stimulus onset and a nose-poke in any incorrect hole

- Reinforcers earned: equal to the number of correct responses

- Anticipatory response rate: the mean number of responses per second during the ITI calculated by using the following formula: total number of responses in all ITI of a session/number of trials/ITI length in seconds.

The accuracy measure was not calculated when the sum of correct and incorrect responses was 15 or less. For analysis of variance (ANOVA), percentage data for accuracy and omissions were arc-sine transformed, latencies were log-transformed and anticipatory response data were subject to square root transformation, as in previous studies (Hahn et al. 2002, 2003a; Patel et al. 2006). For post hoc tests, the Bonferroni correction for multiple comparisons was applied to $t$ tests. Graphs show untransformed data. Results for each measure were analysed by ANOVA for repeated measures using Unistat 5.0 for Excel, three-factor
ANOVA was used to examine data from experiment 1a and two-factor ANOVA was used for experiments $1 b, 2 a$ and $2 b$. Further details of the factors are given below, at relevant places in the Results section.

Drugs

Nicotine hydrogen tartrate (Sigma, UK) was dissolved in isotonic saline with $\mathrm{pH}$ adjusted to 7 with $\mathrm{NaOH}$ solution. All injections were given s.c. in the flank in a volume of $1 \mathrm{ml} / 100 \mathrm{~g}$ and all doses were those of the nicotine base.

\section{Results}

Experiment 1a: initial acquisition of attentional task

There were no significant differences between any of the groups with respect to any parameter of performance at the first stage of training with the stimulus duration set at $60 \mathrm{~s}$ and the ITI at $2 \mathrm{~s}$. Figure 1a) shows the similarity in the changes in accuracy for $\alpha 7^{-1-}$ and wild-type mice over these first seven sessions. It can be seen that the data show signs that the mice began to learn the task from the very earliest stage of training. Results for other variables are not shown because they were similarly negative with respect to genotype.

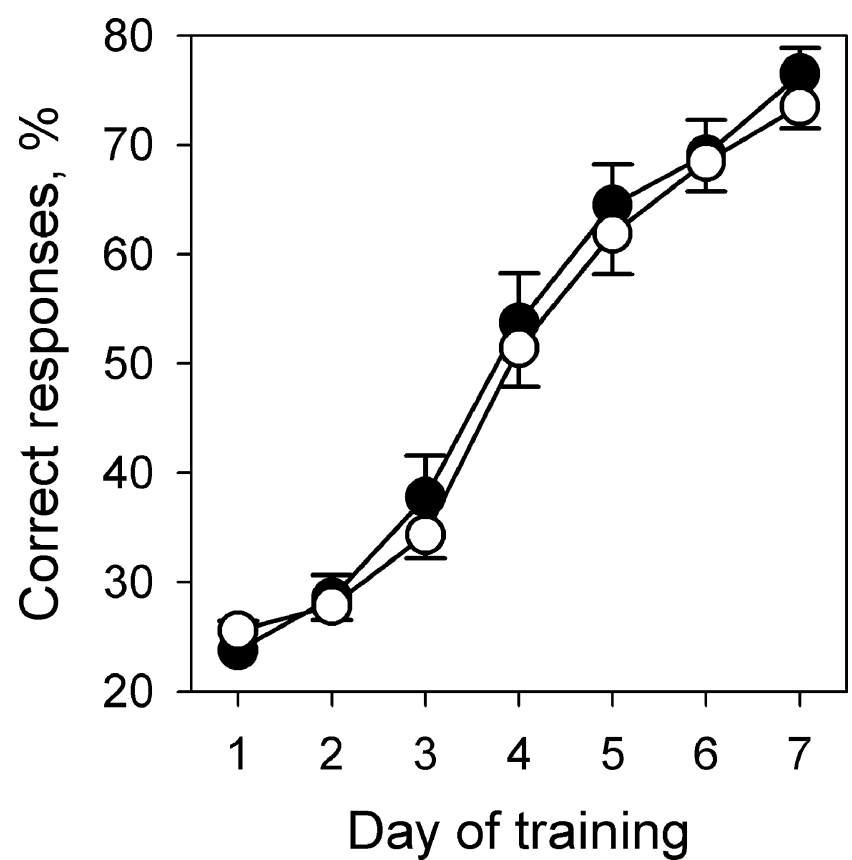

Fig. 1 Experiment 1a: accuracy of 5-CSRTT performance for wildtype $(\bigcirc)$ and $\alpha 7^{-1-}$ mice $(O)$ during the first seven sessions of training in the 5-CSRTT. Results show main effects of genotype and training day as means \pm SEM for groups of 23 mice. Subgroups of mice with each genotype were trained with fixed and variable inter-trial intervals without effect on performance (data not shown) 
During subsequent stages of training there were progressively greater demands made upon the animals' ability by altering task parameters, such as reducing the stimulus duration (Table 1). Differences between the genotypes then emerged, including changes in accuracy, response latencies and anticipatory responding. The data for intermediate stages of training are not shown for brevity; they largely replicated the observations made when the final set of task parameters was in operation. The performance of the animals was relatively stable during the last 10 days of training under the final parameters and these data were selected for analysis and are presented next.

Data for the final 10 days of training were examined by three-factor analysis of variance, the factors being genotype, ITI mode, and days. This analysis yielded main effects of genotype on accuracy $[F(1,42)=8.67, p<0.01]$, reinforcers earned $[F(1,42)=12.3, p<0.01]$, anticipatory response rate $[F(1,42)=4.50, p<0.05]$ and arguably, on correct response latency $[F(1,42)=3.96, p=0.053]$. Figure 2a shows that $\alpha 7^{-/-}$mice were much less accurate
Fig. 2 Experiment 1a: main effects of genotype and day of training on performance of wildtype (O) and $\alpha 7^{-/-}$mice $(O)$ during the last 10 days before drug testing began $(n=23)$. Subgroups of mice of each genotype were trained in fixed $(\mathrm{F})$ and variable $(\mathrm{V})$ inter-trial interval modes; inset bar graphs illustrate the significant main effects of this variable. All data shown are means $\pm \operatorname{SEM}(n=23)$
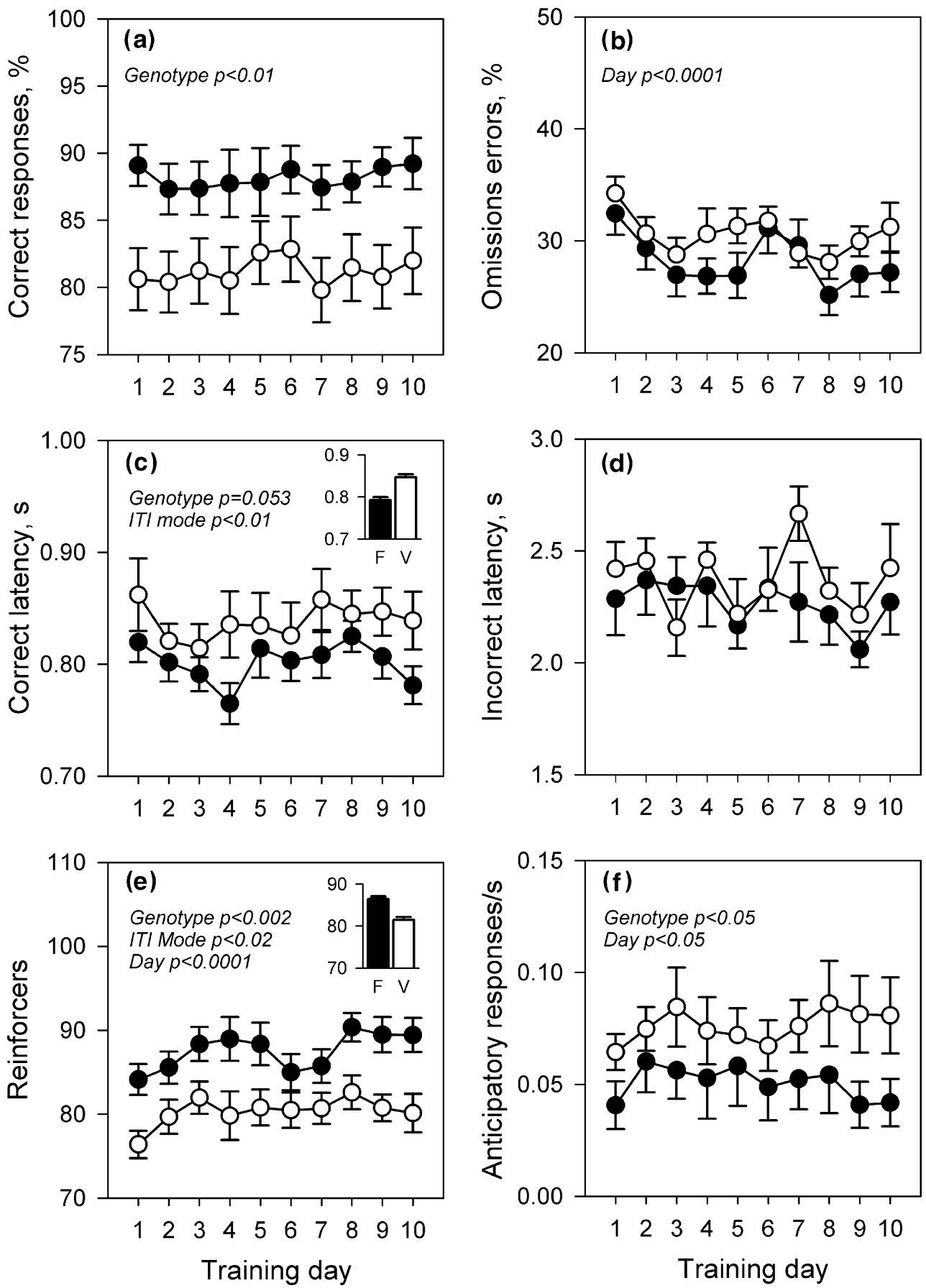
than wild-types, were slightly slower to make a correct response, earned fewer reinforcers and responded more frequently during the ITI. Neither the three-way (group $\times$ ITI mode $\times$ day) nor any two-way interaction was significant for any of the parameters measured. For changes across the ten successive days of training, omission errors $[F(9,378)=4.99, p<0.0001]$, reinforcers earned $[F(9,378)=$ $3.28, p<0.001]$ and anticipatory response rate $[F(9,378)=$ 2.11, $p<0.05]$ showed significant effects. Most of these effects did not show consistent patterns over days (Fig. 2) and are not considered further.

For ITI mode, there were significant main effects for two variables, correct response latency $[F(1,42)=9.27, p<0.01]$ and reinforcers earned $[F(1,42)=6.25, p<0.02]$, with those mice trained on variable ITI mode being slower to make correct responses and obtaining fewer reinforcers. The inset bar graphs in Fig. 2c and e illustrate the significant main effects of ITI mode.

Experiment 1b: first set of nicotine tests

Data were examined with two-factor analysis of variance, the factors being genotype and drug (nicotine) dose. There were no significant effects of nicotine $(0.001-0.1 \mathrm{mg} / \mathrm{kg})$ upon any measure of performance [largest $F(3,78)=2.32$, $p=0.082$, for correct response latency]. The main effect of genotype was significant for accuracy $[\mathrm{F}(1,26)=4.42$, $p<0.05]$ and reinforcers earned $[F(1,26)=6.55, p<0.02]$. Table 2 shows means \pm SEM. for both wild-type and $\alpha 7^{-/}$ mice, for each attentional parameter, over the 30-min sessions. As in experiment 1a, $\alpha 7^{-1-}$ mice were less accurate than wild-types and earned fewer reinforcers.
Genotypes did not differ significantly for other variables, although inspection of Table 2 reveals trends for omission errors, correct response latencies and anticipatory response rate that were in the same direction as the effects seen in experiment 1a. There were no significant interactions between genotype and nicotine.

Experiment 2a: retraining after changing parameters

After the parameter changes listed in Table 1, animals were retrained on the task for several weeks and the relatively stable performance during the last ten sessions of training before tests with nicotine was selected for analysis. Data were examined by means of two-factor analysis of variance, the factors being genotype and day of training. The analysis yielded significant effects of genotype on omission errors $[F(1,29)=20.4, p<0.001]$ and reinforcers earned $[F(1,29)=$ 19.7, $p<0.001]$, but not on accuracy, correct or incorrect response latency or anticipatory response rate [maximum $F(1,29)=1.73]$. The $\alpha 7^{-/-}$mice omitted responses to a greater percentage of stimuli and earned fewer reinforcers than wild-types; these effects were clearly manifested during each of the 10 days of training (Fig. 3b,e).

There were also small but significant differences between correct response latencies and anticipatory responding over days. Anticipatory responding occurred at a lower rate in the later stages of training $[F(9,241)=7.07$, $p<0.0001]$; the latency of correct responses also varied over days, but no clear pattern was evident $[F(9,241)=2.12$, $p<0.05]$. The day of training had no effect on any other parameter. There were no significant genotype $\times$ day interactions.

Table 2 Mean \pm SEM effects of nicotine $(0.001-0.1 \mathrm{mg} / \mathrm{kg}$ ) on attentional parameters in the 5-CSRTT in $\alpha 7$ knockout (KO) mice and their wildtype (WT) littermates

\begin{tabular}{|c|c|c|c|c|c|c|}
\hline \multicolumn{7}{|c|}{ Attentional parameters } \\
\hline \multirow[t]{2}{*}{ Nicotine (mg/kg) } & \multicolumn{2}{|c|}{ Accuracy (percent correct) } & \multicolumn{2}{|c|}{ Omission errors (percent omissions) } & \multicolumn{2}{|c|}{ Correct response latency (s) } \\
\hline & $W T$ & $K O$ & $W T$ & KO & $W T$ & $K O$ \\
\hline 0.0 & $88.9 \pm 1.7$ & $84.7 \pm 2.3$ & $27.4 \pm 2.8$ & $30.5 \pm 1.8$ & $0.79 \pm 0.03$ & $0.85 \pm 0.03$ \\
\hline 0.001 & $89.7 \pm 1.3$ & $84.2 \pm 2.7$ & $25.7 \pm 2.2$ & $30.3 \pm 1.7$ & $0.78 \pm 0.02$ & $0.84 \pm 0.04$ \\
\hline 0.01 & $88.0 \pm 2.1$ & $81.7 \pm 2.5$ & $27.4 \pm 2.3$ & $31.5 \pm 2.4$ & $0.80 \pm 0.02$ & $0.82 \pm 0.02$ \\
\hline \multirow[t]{3}{*}{0.10} & $88.8 \pm 2.0$ & $83.2 \pm 1.8$ & $26.1 \pm 2.5$ & $29.6 \pm 2.4$ & $0.81 \pm 0.02$ & $0.89 \pm 0.03$ \\
\hline & \multicolumn{2}{|c|}{ Incorrect response latency (s) } & \multicolumn{2}{|c|}{ Reinforcers earned (30 min) } & \multicolumn{2}{|c|}{ Anticipatory responses/s } \\
\hline & $W T$ & $K O$ & $W T$ & $K O$ & $W T$ & $K O$ \\
\hline 0.0 & $2.27 \pm 0.19$ & $2.25 \pm 0.22$ & $89.0 \pm 2.9$ & $82.7 \pm 2.5$ & $0.042 \pm 0.010$ & $0.052 \pm 0.010$ \\
\hline 0.001 & $2.05 \pm 0.24$ & $2.45 \pm 0.11$ & $90.8 \pm 2.5$ & $82.9 \pm 3.0$ & $0.038 \pm 0.008$ & $0.059 \pm 0.010$ \\
\hline 0.01 & $2.32 \pm 0.16$ & $2.16 \pm 0.14$ & $88.1 \pm 2.0$ & $80.3 \pm 2.7$ & $0.045 \pm 0.013$ & $0.061 \pm 0.013$ \\
\hline 0.10 & $2.32 \pm 0.20$ & $2.41 \pm 0.15$ & $90.1 \pm 2.9$ & $82.6 \pm 2.3$ & $0.041 \pm 0.011$ & $0.063 \pm 0.013$ \\
\hline
\end{tabular}


Fig. 3 Experiment 2a: main effects of genotype and training day on performance of wild-type (O) and $\alpha 7^{-/-}$mice $(O)$ after reduction of limited hold from 5 to $2 \mathrm{~s}$ and introduction of $2-\mathrm{s}$ time-out for anticipatory responding (all mice trained in variable ITI mode). Results shown are for the last 10 days of training with the preceding altered parameters before the second set of tests with nicotine. Data shown are means \pm SEM $(n=14-15)$
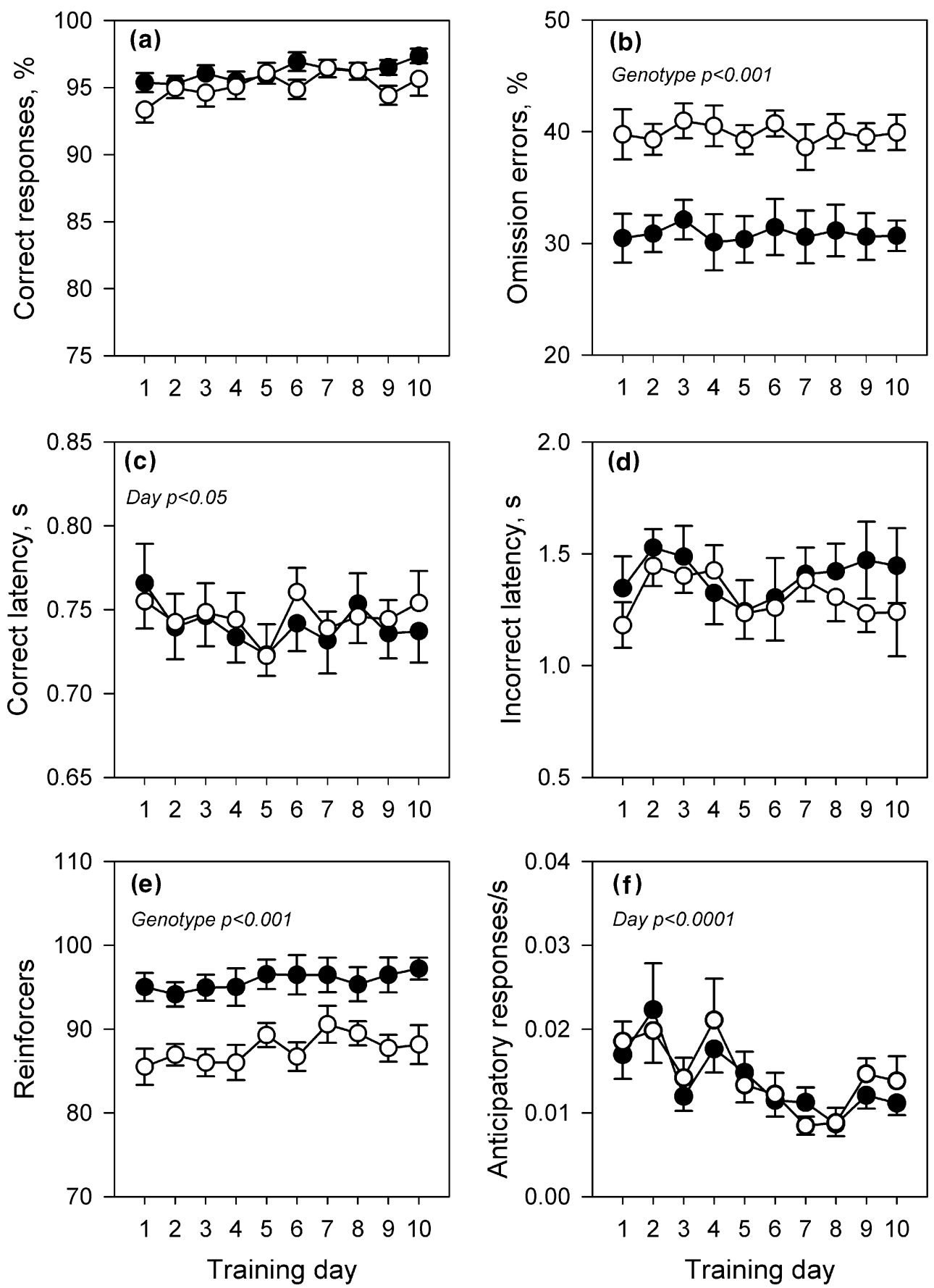

Experiment 2b: second set of nicotine tests

These data were examined by two-factor analysis of variance, the factors being genotype and drug (dose of nicotine). There were no effects of genotype $[F(1,27)=$ $2.79]$ or drug $[F(7,179)=1.44]$ on the percentage of correct responses; nor was there a significant group $\times$ genotype interaction $[F(7,179)=0.97]$. For omission errors there was a significant effect of genotype $[F(1,27)=16.7, p<0.0001]$ and of drug $[F(7,189)=82.4, p<0.0001]$ and additionally, a drug $\times$ group interaction was observed $[F(7,189)=6.40$, $p<0.0001]$. From Fig. $4 \mathrm{~b}$ it can be seen that the effect of genotype arises from the slightly larger overall number of omission errors made by the $\alpha 7^{-/-}$mice. In view of the significant interaction, this difference was examined separately at each dose of nicotine by means of $t$ tests with Bonferroni corrections for multiple comparisons. These analyses yielded significant differences between the genotypes at nicotine doses of $0.003,0.01,0.1$ and $1.0 \mathrm{mg} / \mathrm{kg}$; in all cases the percentage of omission errors was greater in 
Fig. 4 Experiment 2b: main effects of genotype and nicotine dose on performance of wildtype $(\bigcirc)$ and $\alpha 7^{-1-}$ mice $(\bigcirc)$ after reduction of limited hold from 5 to $2 \mathrm{~s}$ and introduction of 2-s time-out for anticipatory responding (all mice trained in variable ITI mode). Data shown are means \pm SEM $(n=14-15)$. Asterisks represent differences between genotypes at each dose of nicotine determined by $t$ tests with Bonferroni correction; $\left.{ }^{*} p<0.05, * * p<0.01\right)$
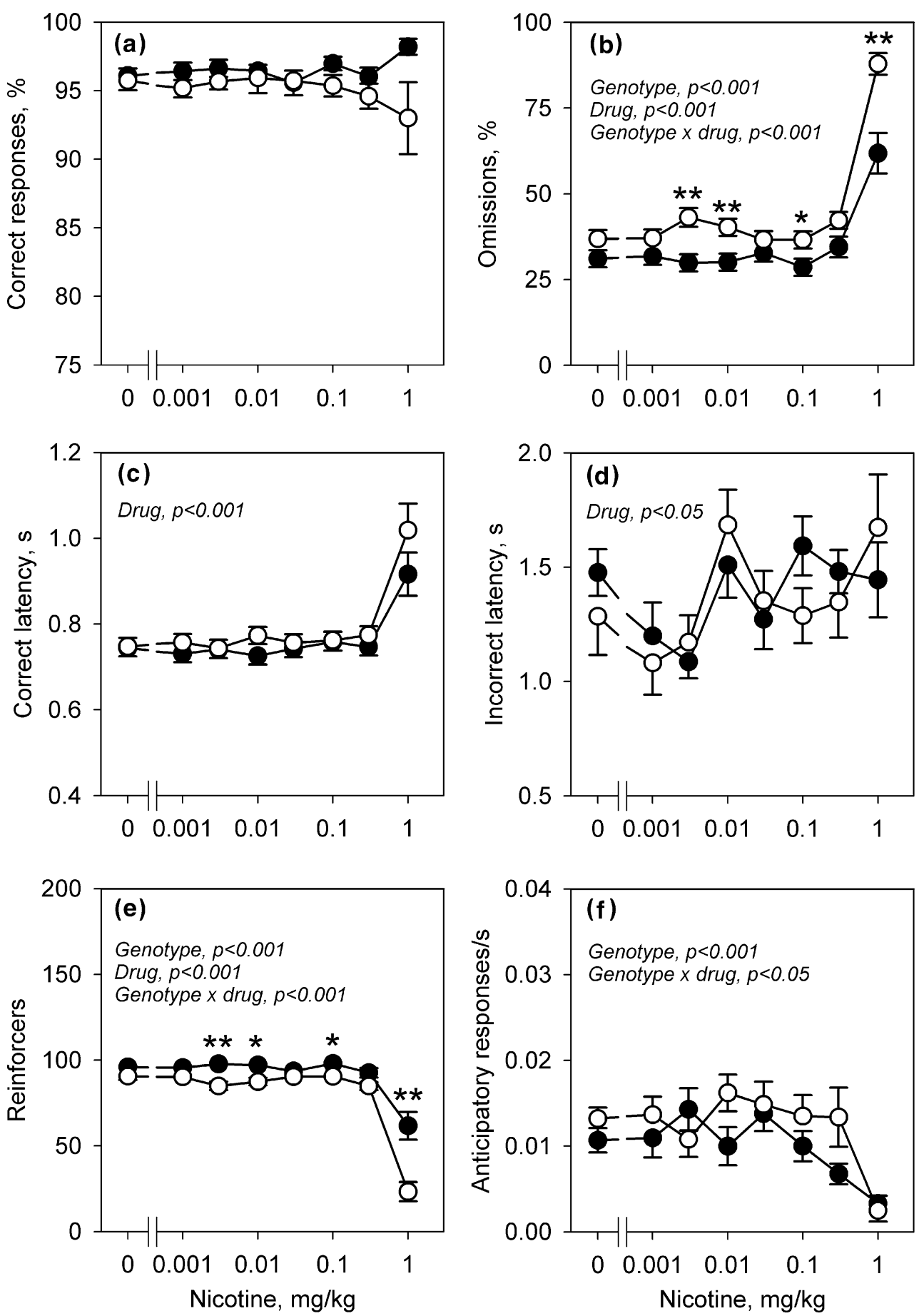

the $\alpha 7^{-/-}$than in the wild-type animals. Nicotine increased omission errors markedly at the largest dose tested, and this effect was greater in the $\alpha 7^{-/-}$than in the wild-type mice.

There was no main effect of genotype on correct latency $[F(1,27)=1.47]$. The main effect of drug on this measure was significant $[F(7,183)=22.4, p<0.0001]$ and this appeared to be entirely due to an increase in latency at the largest dose of nicotine (Fig. 4c). The interaction was not significant $[F(7,183)=1.63]$. There was also a significant effect of drug on incorrect latencies $[F(7,166)=2.23$, $p<0.05$ ), although its origin is not obvious from Fig. $4 \mathrm{~d}$ and there was no dose-response relationship.

With respect to reinforcers earned, there were significant effects of genotype $[F(1,27)=20.7, p<0.0001]$, drug $[F(7$, $189)=82.0, p<0.0001]$ and a group $\times$ drug interaction $[F(7$, $189)=8.71, p<0.0001]$. The overall number of reinforcers earned was slightly greater in wild-type than in $\alpha 7^{-/-}$mice, and Fig. 4e shows that this difference was significant at four 
of the eight dose levels of nicotine that were tested. Nicotine itself decreased the number of reinforcers at the largest dose tested and this effect was greater in $\alpha 7^{-/-}$than in wild-type mice. Finally, there was a significant effect of nicotine $[F(7$, $189)=15.8, p<0.0001]$ but not genotype $[F(1,27)=1.10]$ on anticipatory responding. The effect of drug may be attributed to a decrease in the rate of responding at the $1.0 \mathrm{mg} / \mathrm{kg}$ dose of nicotine (Fig. 4f). A significant drug $\times$ genotype interaction was seen $[F(7,189)=2.39, p<0.05]$, but post hoc tests did not identify a significant difference between the genotypes at any dose level of nicotine.

\section{Discussion}

The five-choice serial reaction time task revealed impairments in the performance of the $\alpha 7$ nicotinic receptor knockout mice on a variety of measures, thus supporting the view that these receptors play a role in attentional performance. Analysis through variation in task parameters suggested that the primary deficit was an increase in omission errors; a general slowing in performance would have been reflected in corresponding increases in response latencies and decreases in anticipatory responding. Thus, the $\alpha 7^{-/-}$mice failed to detect significantly more stimuli than the wild-types, but when they did detect a stimulus they responded to it quickly and correctly. These findings confirm and extend the key observations of Young et al. (2004) and support the emerging consensus that although $\alpha 7^{-/-}$mice show deficits in the performance of several cognitive tasks, the differences from controls are modest and are manifest only with specific tasks used under precisely defined conditions (Paylor et al. 1998; Wehner et al. 2004; Fernandes et al. 2005). We now discuss in detail some differences between our results and those of Young et al. (2004) and indicate how they can be reconciled by considering parametric variations in the procedures used.

In the present study, $\alpha 7^{-/-}$mice did not differ from wild types until the later stages of training, where the more stringent task parameters placed greater demands upon their cognitive ability (Fig. 2). These differences in the baseline (undrugged) performance of the $\alpha 7^{-/-}$mice and wild-type mice were largely replicated in our first study on the response to nicotine (Fig. 3). The impaired accuracy and increased response latency suggested an attentional impairment, whereas the increase in anticipatory responding suggested greater impulsivity. Keller et al. (2005) also found that the amount of anticipatory responding was increased in $\alpha 7^{-1-}$ mice, although they used a different behavioural procedure.

There is often an inverse relationship between accuracy and impulsivity in the 5-CSRTT, and from a comparison of inbred mouse strains it was argued that a decrease in accuracy could be secondary to increased impulsivity (Patel et al. 2006). The observed change in impulsivity may have contributed to the decrease in accuracy in experiment 1a, which should, therefore, be interpreted cautiously. We did not observe slow acquisition of task performance like that seen by Young et al. (2004); a primary effect on anticipatory responding may have contributed to their finding, too, rather than a deficit in learning ability. This interpretation is supported by the absence of impairments in $\alpha 7^{-/-}$mice in a variety of other learning tasks (Paylor et al. 1998) and is especially likely with the definition of accuracy used by Young et al. (2004), which included the number of anticipatory responses in the denominator for calculation of the proportion of correct responses.

During experiment 1a we did not observe any difference between $\alpha 7^{-/-}$and wild-type mice with respect to omission errors. Interestingly, Young et al. (2004) reported an increase in omission errors in the $\alpha 7^{-1-}$ mice. The difference in durations of the limited holds (LH) used in the two studies may explain this apparent disagreement. The LH, the period in which responses are recorded as being correct or incorrect following stimulus termination, was $2 \mathrm{~s}$ in Young et al. (2004) and $5 \mathrm{~s}$ in the final stage of experiment 1a. When, with a long $\mathrm{LH}$, a stimulus is not detected, 'impulsive' responses in the period of the LH are more likely to be recorded as incorrect than as correct responses, as there are four times more incorrect response options than there are correct options. The increased impulsivity observed in $\alpha 7^{-/-}$mice may, therefore, have contributed to the observed reduction in accuracy.

At the same time, an increase in omission errors such as that reported by Young et al. (2004) may have been masked, as training with a long limited hold gives the animals more time to make an impulsive response. The trend $(p<0.06)$ towards longer correct response latencies in $\alpha 7^{-/-}$mice in experiment 1a might also be explained by late 'correct' impulsive responses rather than slower reactions. Both groups had short response latencies and the differences between them were eliminated when impulsive responses were punished and the LH was reduced in experiment 2a. Additional and more detailed data on the temporal and spatial patterning of errors and systematic studies of the effects of punishment and of varying LH is needed to establish the validity of this interpretation.

In an attempt to understand the origin of the differences between the present results and those of Young et al. (2004), we retrained the mice with the LH reduced from 5 to $2 \mathrm{~s}$, used only variable ITI and introduced a 2 -s time-out for anticipatory responses; these task parameters resembled closely those of Young et al. (2004). In experiment 2a, carried out under these altered conditions, there was a clear increase in omission errors in the $\alpha 7^{-/-}$mice. In contrast, 
the percentage of correct responses was similar in wild-type and $\alpha 7^{-1-}$ animals and the previously observed differences in the latencies of correct responses and in anticipatory response rates were no longer seen. This pattern of deficits in $\alpha 7^{-/-}$mice was indistinguishable from that observed by Young et al. (2004).

The absence of a difference in anticipatory responding between genotypes may have been associated with the reduction in its rate as a consequence of the time-out contingency in experiment $2 \mathrm{a}$; the use of variable ITI in the retraining phase may also have been important because the initial difference between the wild-type and $\alpha 7^{-/-}$mice in anticipatory responding was greater in animals trained with fixed interval than with variable interval (Fig. 2). This observation might reflect impaired utilisation of temporally predictable stimuli in the $\alpha 7^{-/-}$mice, rather than differences in impulsivity per se. Nevertheless, caution is needed because the altered performance after parametric changes may also have been associated with the longer training and greater age of the animals at this stage.

In experiment $1 \mathrm{~b}$, the absence of any significant effect of nicotine even in wild-type mice could have been attributed to the parameters of the schedule maintaining the behaviour. Nevertheless, the doses of nicotine used were within the range reported to improve accuracy and reduce omission errors in mice (Young et al. 2004). It, therefore, seemed possible that a nicotine effect would emerge when the training parameters were adjusted to resemble those used by Young et al. (2004). However, this was not the case and in experiment $2 \mathrm{~b}$; no dose of nicotine improved performance on any variable. The largest dose of nicotine impaired performance; omission errors and the latencies of correct responses increased, whereas reinforcers earned and anticipatory responses decreased (Fig. 4f). This pattern of results can be attributed to a general deficit in operant response rates produced by the very large $(1 \mathrm{mg} / \mathrm{kg})$ dose of nicotine. The lack of change in accuracy is compatible with explanations in terms of motor impairment rather than an attentional deficit. The data in Fig. $4 \mathrm{a}$ indicate that these effects of nicotine were also present in the $\alpha 7^{-/-}$mice and, in some cases, their magnitude was increased (e.g. omission errors). The reason for this unexpected observation is unclear; it may represent a little more than a minor enhancement at certain doses of nicotine of the significant main effect of genotype on omission errors.

Why was there no improvement in attentional performance by any dose of nicotine? A ceiling effect associated with the very high baseline accuracy offers only a partial explanation because the baseline rate of omission errors was high $(30 \%)$, but there was still no nicotine-induced improvement on either measure. Prior exposure to nicotine might be relevant, but Young et al. (2004) obtained positive effects of nicotine at 0.003 and $0.3 \mathrm{mg} / \mathrm{kg}$ in mice previously exposed to the drug and in rats, nicotine-induced attentional enhancement did not show tolerance during 6 weeks of daily exposure to the drug (Hahn and Stolerman 2002). The parameters for training and testing during experiment $2 \mathrm{~b}$ were also very similar to those used by Young et al. (2004). On the basis of the available information it appears that nicotine-induced improvement of performance in wild-type mice with a C57BL/6 background is not a robust phenomenon.

This aspect of the study contrasts with the good agreement of the present results with those of Young et al. (2004) with respect to the performance of $\alpha 7^{-/-}$and wildtype mice in experiments 1a and $2 \mathrm{a}$. Comparisons between C57BL/6 and DBA/2 mice in the 5-CSRTT have also been carried out in two different laboratories, and there was excellent agreement between the results; in each case, the $\mathrm{DBA} / 2$ mice were the poorer performers, exhibiting increased impulsivity and lower accuracy (Greco et al. 2005; Patel et al. 2006). DBA/2 mice had reduced levels of $\alpha 7$ expression and impaired sensory gating that was corrected by $\alpha 7$ agonists (Stevens et al. 1998).

The present work did not investigate the mechanisms underlying the impaired performance of $\alpha 7^{-/-}$mice, but some suggestions can be made. Investigations of brain areas important for attentional performance using localised lesions and drug administration have demonstrated the involvement of the medial prefrontal cortex and D1 dopamine receptor activation within this area (Muir et al. 1996; Granon et al. 2000; Hahn et al. 2003b). Dopamine utilisation in the frontal cortex also appears to be reduced in individual rats that perform badly in the task (Puumala and Sirvio 1998), whereas the $\alpha 7$ agonist GTS-21 elevated dopamine and noradrenaline concentrations in the frontoparietal cortex, which is implicated in attentional function (Summers et al. 1997); it is possible that a reduction or lack of $\alpha 7$ receptors might lead to a deficit in dopamine levels in the fronto-parietal cortex, although changes in neurotransmitter systems other than dopamine may also contribute.

It can be suggested, speculatively, that the $\alpha 7^{-/}$mouse may have some value as a model for very specific aspects of the cognitive dysfunctions in schizophrenia. Diminished frontal dopamine has been hypothesised to be important in schizophrenia (Davids et al. 2003; Laruelle et al. 2003), and post-mortem studies have shown diminished numbers of $\alpha 7$ receptors in brains of schizophrenics. The $\alpha 7^{-1-}$ mouse has not been tested for an auditory sensory gating deficit, the endophenotype of schizophrenia in which the $\alpha 7$ nicotinic receptor has been strongly implicated (Freedman et al. 2002) and which may offer a cognitive explanation for the attentional deficits observed.

In conclusion, this study confirms that $\alpha 7^{-/-}$mice exhibit a weakened ability to detect visual stimuli in a test of sustained attention, suggesting that the presence of $\alpha 7$ receptors is 
necessary for normal task performance. Manipulating task parameters can alter the apparent nature of the deficit, suggesting a need to compare different strains of mice under more than one set of conditions to characterise them adequately. While this observation encourages further investigation of the value of $\alpha 7$ receptor agonists in neuropsychiatric states such as dementia (Kitagawa et al. 2003) and schizophrenia (Martin et al. 2004), the lack of effect with nicotine in the present experiments is a major concern. Identification of a protocol for assessing attention-enhancing effects of nicotine in mice may require further modifications of protocols or the use of different strains of animal.

Acknowledgement We thank MRC for financial support through a programme grant.

\section{References}

Berg DK, Conroy WG (2002) Nicotinic alpha 7 receptors: synaptic options and downstream signaling in neurons. J Neurobiol 53:512-523

Bizarro L, Patel S, Murtagh C, Stolerman IP (2004) Differential effects of psychomotor stimulants on attentional performance in rats: nicotine, amphetamine, caffeine and methylphenidate. Behav Pharmacol 15:195-206

Blondel A, Sanger DJ, Moser PC (2000) Characterisation of the effects of nicotine in the five-choice serial reaction time task in rats: antagonist studies. Psychopharmacology 149:293-305

Briggs CA, Anderson DJ, Brioni JD, Buccafusco JJ, Buckley MJ, Campbell JE, Decker MW, Donnelly-Roberts D, Elliott RL, Gopalakrishnan M, Holladay MW, Hui YH, Jackson WJ, Kim DJ, Marsh KC, O’Neill A, Prendergast MA, Ryther KB, Sullivan JP, Arneric SP (1997) Functional characterization of the novel neuronal nicotinic acetylcholine receptor ligand GTS-21 in vitro and in vivo. Pharmacol Biochem Behav 57:231-241

Davids E, Zhang K, Tarazi FI, Baldessarini RJ (2003) Animal models of attention-deficit hyperactivity disorder. Brain Res Brain Res Rev 42:1-21

Fernandes C, Hoyle E, Dempster E, Schalkwyk L, Collier DA (2005) Performance deficit of $\alpha 7$ nicotinic receptor knockout mice in a delayed matching-to-place (DMP) task suggests a mild impairment of working/episodic-like memory. Genes Brain Behav 5:433-440

Franceschini D, Paylor R, Broide R, Salas R, Bassetto L, Gotti C, De Biasi M (2002) Absence of alpha7-containing neuronal nicotinic acetylcholine receptors does not prevent nicotineinduced seizures. Brain Res Mol Brain Res 98:29-40

Freedman R, Hall M, Adler LE, Leonard S (1995) Evidence in postmortem brain tissue for decreased numbers of hippocampal nicotinic receptors in schizophrenia. Biol Psychiatry 38:22-33

Freedman R, Adler LE, Olincy A, Waldo MC, Ross RG, Stevens KE, Leonard S (2002) Input dysfunction, schizotypy, and genetic models of schizophrenia. Schizophr Res 54:25-32

Granon S, Passetti F, Thomas KL, Dalley JW, Everitt BJ, Robbins TW (2000) Enhanced and impaired attentional performance after infusion of D1 dopaminergic receptor agents into rat prefrontal cortex. J Neurosci 20:1208-1215

Greco B, Invernizzi RW, Carli M (2005) Phencyclidine-induced impairment in attention and response control depends on the background genotype of mice: reversal by the mGLU(2/3) receptor agonist LY379268. Psychopharmacology 179:68-76
Grottick AJ, Higgins GA (2000) Effect of subtype selective nicotinic compounds on attention as assessed by the five-choice serial reaction time task. Behav Brain Res 117:197-208

Hahn B, Stolerman IP (2002) Nicotine-induced attentional enhancement in rats: effects of chronic exposure to nicotine. Neuropsychopharmacology 27:712-722

Hahn B, Shoaib M, Stolerman IP (2002) Nicotine-induced enhancement of attention in the five-choice serial reaction time task: the influence of task demands. Psychopharmacology 162:129-137

Hahn B, Sharples CG, Wonnacott S, Shoaib M, Stolerman IP (2003a) Attentional effects of nicotinic agonists in rats. Neuropharmacology 44:1054-1067

Hahn B, Shoaib M, Stolerman IP (2003b) Involvement of the prefrontal cortex but not the dorsal hippocampus in the attention-enhancing effects of nicotine in rats. Psychopharmacology 168:271-279

Humby T, Laird FM, Davies W, Wilkinson LS (1999) Visuospatial attentional functioning in mice: interactions between cholinergic manipulations and genotype. Eur J Neurosci 11:2813-2823

Keller JJ, Keller AB, Bowers BJ, Wehner JM (2005) Performance of alpha7 nicotinic receptor null mutants is impaired in appetitive learning measured in a signaled nose poke task. Behav Brain Res 162:143-152

Kitagawa H, Takenouchi T, Azuma R, Wesnes KA, Kramer WG, Clody DE, Burnett AL (2003) Safety, pharmacokinetics, and effects on cognitive function of multiple doses of GTS-21 in healthy, male volunteers. Neuropsychopharmacology 28:542-551

Laruelle M, Kegeles LS, Abi-Dargham A (2003) Glutamate, dopamine, and schizophrenia: from pathophysiology to treatment. Ann NY Acad Sci 1003:138-158

Martin LF, Kem WR, Freedman R (2004) Alpha-7 nicotinic receptor agonists: potential new candidates for the treatment of schizophrenia. Psychopharmacology 174:54-64

Marutle A, Zhang X, Court J, Piggott M, Johnson M, Perry R, Perry E, Nordberg A (2001) Laminar distribution of nicotinic receptor subtypes in cortical regions in schizophrenia. J Chem Neuroanat 22:115-126

Muir JL, Everitt BJ, Robbins TW (1996) The cerebral cortex of the rat and visual attentional function: dissociable effects of mediofrontal, cingulate, anterior dorsolateral, and parietal cortex lesions on a five-choice serial reaction time task. Cereb Cortex 6:470-481

Naylor C, Quarta D, Fernandes C, Stolerman IP (2005) Tolerance to nicotine in mice lacking alpha7 nicotinic receptors. Psychopharmacology 180:558-563

Olivier B, Leahy C, Mullen T, Paylor R, Groppi VE, Sarnyai Z, Brunner D (2001) The DBA/2J strain and prepulse inhibition of startle: a model system to test antipsychotics? Psychopharmacology $156: 284-290$

O’Neill HC, Rieger K, Kem WR, Stevens KE (2003) DMXB, an alpha7 nicotinic agonist, normalizes auditory gating in isolationreared rats. Psychopharmacology 169:332-339

Orr-Urtreger A, Goldner FM, Saeki M, Lorenzo I, Goldberg L, De Biasi M, Dani JA, Patrick JW, Beaudet AL (1997) Mice deficient in the alpha7 neuronal nicotinic acetylcholine receptor lack alpha-bungarotoxin binding sites and hippocampal fast nicotinic currents. J Neurosci 17:9165-9171

Patel S, Stolerman IP, Asherson P, Sluyter F (2006) Attentional performance of $\mathrm{C} 57 \mathrm{BL} / 6$ and $\mathrm{DBA} / 2$ mice in the 5-choice serial reaction time task. Behav Brain Res 170:197-203

Paylor R, Nguyen M, Crawley JN, Patrick J, Beaudet A, Orr-Urtreger A (1998) Alpha7 nicotinic receptor subunits are not necessary for hippocampal-dependent learning or sensorimotor gating: a behavioral characterization of Acra7-deficient mice. Learn Mem 5:302-316

Puumala T, Sirvio J (1998) Changes in activities of dopamine and serotonin systems in the frontal cortex underlie poor choice 
accuracy and impulsivity of rats in an attention task. Neuroscience 83:489-499

Schreiber R, Dalmus M, De Vry J (2002) Effects of alpha 4/beta 2and alpha7-nicotine acetylcholine receptor agonists on prepulse inhibition of the acoustic startle response in rats and mice. Psychopharmacology 159:248-257

Seguela P, Wadiche J, Dineley-Miller K, Dani JA, Patrick JW (1993) Molecular cloning, functional properties, and distribution of rat brain alpha7: a nicotinic cation channel highly permeable to calcium. J Neurosci 13:596-604

Stevens KE, Kem WR, Mahnir VM, Freedman R (1998) Selective alpha7-nicotinic agonists normalize inhibition of auditory response in DBA mice. Psychopharmacology 136:320-327

Stolerman IP, Chamberlain S, Bizarro L, Fernandes C, Schalkwyk L (2004) The role of nicotinic receptor alpha 7 subunits in nicotine discrimination. Neuropharmacology 46:363-371

Summers KL, Kem WR, Giacobini E (1997) Nicotinic agonist modulation of neurotransmitter levels in the rat frontoparietal cortex. Jpn J Pharmacol 74:139-146
Tritto T, McCallum SE, Waddle SA, Hutton SR, Paylor R, Collins AC, Marks MJ (2004) Null mutant analysis of responses to nicotine: deletion of beta2 nicotinic acetylcholine receptor subunit but not alpha7 subunit reduces sensitivity to nicotineinduced locomotor depression and hypothermia. Nicotine Tob Res 6:145-158

Waldo MC, Carey G, Myles-Worsley M, Cawthra E, Adler LE, Nagamoto HT, Wender P, Byerley W, Plaetke R, Freedman R (1991) Codistribution of a sensory gating deficit and schizophrenia in multi-affected families. Psychiatry Res 39:257-268

Wehner JM, Keller JJ, Keller AB, Picciotto MR, Paylor R, Booker TK, Beaudet A, Heinemann SF, Balogh SA (2004) Role of neuronal nicotinic receptors in the effects of nicotine and ethanol on contextual fear conditioning. Neuroscience 129: $11-24$

Young JW, Finlayson K, Spratt C, Marston HM, Crawford N, Kelly JS, Sharkey J (2004) Nicotine improves sustained attention in mice: evidence for involvement of the alpha7 nicotinic acetylcholine receptor. Neuropsychopharmacology 29:891-900 